\title{
How to identify indolent thyroid tumors unlikely to recur and cause cancer death immediately after surgery-Risk stratification of papillary thyroid carcinoma in young patients-
}

\author{
Kennichi Kakudo ${ }^{1)}$, Zhiyan $\mathrm{Liu}^{2)}$, Yanhua Bai ${ }^{3)}$, Yaqiong $\mathrm{Li}^{4}$, , Naomi Kitayama ${ }^{5)}$, Shinya Satoh ${ }^{6}$, \\ Masahiro Nakashima ${ }^{7)}$ and Chan Kwon Jung ${ }^{8)}$ \\ 1) Department of Pathology, Cancer Genome Center and Thyroid Disease Center, Izumi City General Hospital, Osaka, Japan \\ 2) Department of Pathology, Shanghai Sixth People's Hospital Affiliated with Shanghai Jiao Tong University School of Medicine, \\ Shanghai, China \\ ${ }^{3)}$ Key Laboratory of Carcinogenesis and Translational Research (Ministry of Education), Department of Pathology, Peking \\ University Cancer Hospital \& Institute, Beijing, China \\ 4) Department of Pathology, Shandong Provincial Hospital Affiliated with Shandong First Medical University, Jinan, China \\ 5) Department of Dermatology, Kyoto University Graduate School of Medicine, Kyoto, Japan \\ 6) Department of Endocrine Surgery, Yamashita Thyroid Hospital, Fukuoka, Japan \\ 7) Department of Tumor and Diagnostic Pathology, Atomic Bomb Disease Institute, Nagasaki University, Nagasaki, Japan \\ 8) Department of Hospital Pathology, College of Medicine, The Catholic University of Korea, Seoul, Korea
}

\begin{abstract}
Current histopathological diagnosis methods cannot distinguish the two types of thyroid carcinoma: clinically significant carcinomas with a potential risk of recurrence, metastasis, and cancer death, and clinically insignificant carcinomas with a slow growth rate. Both thyroid tumors are diagnosed as "carcinoma" in current pathology practice. The clinician usually recommends surgery to the patient and the patient often accepts it because of cancer terminology. The treatment for these clinically insignificant carcinomas does not benefit the patient and negatively impacts society. The author proposed risk stratification of thyroid tumors using the growth rate (Ki-67 labeling index), which accurately differentiates four prognostically relevant risk groups based on the Ki-67 labeling index, $\geq 30 \%, \geq 10$ and $<30 \%,>5$ and $<10 \%$, and $\leq 5 \%$. Indolent thyroid tumors with an excellent prognosis have the following four features: young age, early-stage (T1-2 M0), curatively treated, and low proliferation index (Ki-67 labeling index of $\leq 5 \%$ ), and are unlikely to recur, metastasize, or cause cancer death. Accurate identification of these indolent tumors helps clinicians select more conservative treatments to avoid unnecessary aggressive (total thyroidectomy followed by radio-active iodine) treatments. Clinicians can alleviate the fears of patients by confirming these four features, including the low proliferation rate, in a pathology report immediately after surgery when patients are most concerned.
\end{abstract}

Key words: Thyroid carcinoma, Prognosis, Ki-67, Patient anxiety, Risk assessment

\section{Introduction}

A marked increase in the prevalence of thyroid carcinoma has been observed worldwide. However, the number of deaths from thyroid carcinoma remains unchanged. Based on these two observations, epidemiologists sug-

Submitted Jan. 8, 2021; Accepted Apr. 16, 2021 as EJ21-0018 Released online in J-STAGE as advance publication May 11, 2021 Correspondence to: Kennichi Kakudo, MD, Ph.D., Department of Pathology, Cancer Genome Center and Thyroid Disease Center, Izumi City General Hospital, Wake-Cho 4-5-1, Izumi-city, Osaka 594-0073, Japan.

E-mail: kakudo@thyroid.jp gested a high risk of overdiagnosis and overtreatment of thyroid carcinomas in current clinical practice, which does not benefit the patient and negatively impacts society as a whole [1-3]. This opinion paper introduces a prognostic risk classification of thyroid tumors using the Ki-67 labeling index $[4,5]$ and a strategy to integrate it with the AJCC (American Joint Committee on Cancer) TNM (tumor-node-metastasis) staging system [6] to identify indolent thyroid tumors that are unlikely to recur, metastasize, or cause cancer death. It reliably distinguishes clinically significant carcinomas with the potential risk of recurrence, metastasis, and cancer death from clinically insignificant carcinomas with a slow 
growth rate. A high Ki-67 labeling index also identifies carcinomas with a high risk of recurrence and death [7-13], and the Ki-67 labeling index was previously recommended as an essential component in pathology reporting $[5,14]$.

\section{Heterogeneity of Cancer Progression Based on the Tumor Growth Speed According to Welch}

Welch classified cancer into four types (fast-growing, slow-growing, very slow-growing, and non-progressive) based on growth speed [1]. Fast-growing cancer develops local and systemic symptoms due to rapid tumor growth and metastasis, and patients die of tumors in a short period after the onset of the disease. Among thyroid carcinomas, anaplastic carcinoma of the thyroid corresponds to fast-growing cancer. Slow-growing cancer grows and manifests over more than several years. If left untreated, the patient will die of the tumor years later. If it can be detected/treated at an early stage, it is curable. Very slow-growing cancer does not cause death and the patient dies of another illness before the tumor causes any symptom. Welch emphasized that very slow-growing cancer does not harm the patient and does not require treatment. The fourth group is cancer that grows but spontaneously disappears and is termed non-progressive cancer. However, many thyroid carcinomas, including symptomatic carcinomas and carcinomas with lymph node metastasis, do not cause death [15-22]. The 10-year disease-specific survival rate (DSSR) of AJCC stage I papillary thyroid carcinoma (PTC) was reported to be $99.6 \%$ by Kim et al. in a Korean patient cohort [19], $99.7 \%$ in a Chinese patient cohort by Tam et al. [18], and $99.6 \%$ in a European population by van Velsen et al. [20]. An international multi-institutional study by Nixon et al. of 9.484 thyroid cancer patients revealed a 10-year DSSR of $99.5 \%$ [21]. The 20-year DSSR rate of AJCC stage I PTC was $99.3 \%$ in a Japanese patient cohort reported by Ito et al. [22]. Therefore, cancer mortality does not occur in more than $99 \%$ of patients with AJCC stage I PTC after complete excision regardless of lymph node metastasis and extrathyroid invasion.

\section{Risk Classification of Thyroid Tumors Based on the Ki-67 Labeling Index}

Incorporating the concept of "tumor growth speed determines the prognosis" advocated by Welch, the author published a risk classification of thyroid follicular cell tumors based on the tumor growth speed in 2015 (Table 1) [4]. This classification is characterized by the Ki-67 labeling index to evaluate the proliferative capacity of tumor cells. Cell growth activity is one of the two characteristic features (abnormal cells divide without control and can invade nearby tissues) of carcinoma [23]. It is an essential feature for assessing the biological behavior of low-grade malignancies. The most useful marker to evaluate cell proliferative activity is Ki-67, which is expressed in the cell nuclei in all cells except for those in the G0 phase [24]. It is immunohistochemically evaluated as the labeling index using formalinfixed paraffin-embedded tissue specimens. There are two potential applications of the Ki-67 labeling index in human neoplasms: 1) differential diagnosis between benign and malignant lesions, and 2) risk classification of low-grade malignancies, which is currently performed in clinical practice for grading diagnoses of neuroendocrine tumors, breast carcinomas, malignant lymphoma, and gastrointestinal stromal tumors [24, 25]. No major staging systems of thyroid tumors (AMES, American Thyroid Association (ATA), EORTIC, Japan Association of Endocrine Surgery (JAES), MACIS, and TNM) include one of the two essential cancer definition criteria, tumor growth speed, in their classification schema. The reason for adopting cutoff values at $3 \%, 5 \%, 10 \%$, and $30 \%$ is because the World Health Organization (WHO) classification previously defined the Ki-67 labeling index of normal follicular cells as $<3 \%$, well-differentiated carcinoma as $<10 \%$, and anaplastic thyroid carcinoma as $>30 \%$ [18]. Furthermore, the cutoff values of $5 \%$ and $10 \%$ are used to maintain consistency with a study from Kuma Hospital by Miyauchi et al., which demonstrated a significant prognostic difference in PTC using these values [12]. Other previous studies reported lower cutoff values $(1.85 \%$ by Kjellman et al., $2.5 \%$ by Tang et al., and $3 \%$ by Ito et al.) $[9,11,13]$. These values were not incorporated in our prognostic classification because they were elucidated from studies with less sensitive AvidinBiotin Complex immunohistochemical methods [4].

As risk stratification of surgically treated cases is highly important in clinical practice, the author modified Welch's original classification for natural history of carcinomas (Table 1). The author excluded non-progressive tumors (shown by a purple dotted line in Fig. 1 for comparison) (Fig. 1). The highest risk group is anaplastic carcinoma. Although the number is small among surgical cases, it was defined as a tumor with a Ki-67 labeling index of $>30 \%$, which corresponds to anaplastic thyroid carcinoma $[4,7,26,27]$. After excluding anaplastic thyroid carcinoma, surgically treated follicular cell-derived thyroid carcinomas were subdivided into the following three (high-, moderate-, and low-) risk groups based on the Ki-67 labeling index, 1) $>10$ and $\leq 30 \%, 2)>5$ and $\leq 10 \%$, and 3 ) $\leq 5 \%$, respectively (Table 1). High-risk thyroid carcinoma of follicular cell origin is a tumor with 
Table 1 Prognostic classification of thyroid tumors using the Ki-67 labeling index [4]

\begin{tabular}{|c|c|c|c|}
\hline Classification of Tumors & Ki-67 & Incidence & Clinical managements \\
\hline \multicolumn{4}{|l|}{1 Benign tumors } \\
\hline Follicular adenoma & $<3 \%$ & na & No further treatment if resected. \\
\hline \multicolumn{4}{|l|}{2 Borderline tumors ( $\mathrm{pN} 0, \mathrm{pEx} 0$ and $\mathrm{M} 0$ ) } \\
\hline \multicolumn{4}{|l|}{ 1) Encapsulated tumors } \\
\hline $\begin{array}{l}\text { HTT, WDT-UMP, FT-UMP, NIFTP, } \\
\text { NEPRAS }\end{array}$ & $<3 \%$ & na & No further treatment if resected. \\
\hline \multicolumn{4}{|l|}{ 2) Non-encapsulated tumors $(<1 \mathrm{~cm})$} \\
\hline Papillary microcarcinoma & $<3 \%$ & na & Active Surveillance or lobectomy. \\
\hline \multicolumn{4}{|l|}{3 Malignant tumors (invasive carcinoma) } \\
\hline 1) Low-risk & $\leq 5 \%$ & $312 / 390(80 \%)$ & Lobectomy is sufficient for stage I PTCs. \\
\hline 2) Moderate-risk & $>5$ and $\leq 10 \%$ & $48 / 390(12 \%)$ & $\begin{array}{l}\text { Multidiciplinary discussion to decide treatment } \\
\text { modality. }\end{array}$ \\
\hline 3) High-risk & $>10$ and $\leq 30 \%$ & $30 / 390(8 \%)$ & Total thyroidectomy with adjuvant RAI. \\
\hline 4) Anaplastic thyroid carcinoma & $>30 \%$ & na & $\begin{array}{l}\text { Systemic treatments after multidiciplinary } \\
\text { discussion. }\end{array}$ \\
\hline
\end{tabular}

The author suggests appropriate clinical management for each risk group in the right-most column. The incidence (second from the right) of PTCs in Ki-67 low-risk, moderate-risk, and high-risk groups treated by total thyroidectomy at Kuma Hospital reported by Miyauchi [12] is shown. HTT, hyalinizing trabecular tumor; WDT-UMP, well-differentiated tumor of uncertain malignant potential; FT-UMP, follicular tumor of uncertain malignant potential; NIFTP, noninvasive follicular thyroid neoplasm with papillary-like nuclear features; NEPRAS, noninvasive encapsulated papillary RAS-like thyroid tumor; RAI, radioactive iodine; na, not applicable.



Fig. 1 Classification of thyroid carcinomas of follicular cell origin in four prognostic risk groups based on the Ki-67 index (proliferation rate) and Welch's non-progressive cancer.

Based on the tumor growth rate (Ki-67 labeling index), the natural history of thyroid carcinomas of follicular cell origin is classified into four risk groups (using cutoff values, $\leq 5 \%,>5$ and $\leq 10 \%,>10 \%$ and $\leq 30 \%$, and $>30 \%$ ). 1) Low-risk (Ki-67 labeling index of $\leq 5 \%$, black arrow), 2) moderate-risk (Ki-67 labeling index of $>5$ and $\leq 10 \%$, blue arrow), 3) high-risk (Ki-67 labeling index of $>10$ and $\leq 30 \%$, green arrow), and 4) anaplastic (Ki-67 labeling index of $>30 \%$, red arrow). Welch's non-progressive cancer was added as a purple dotted line for comparison. Both non-progressive cancer and low-risk carcinoma do not cause cancer death because of their very slow growth rate. In addition to very slow-growing cancer in Welch's classification, the Ki-67 low-risk carcinomas are symptomatic, and may develop small-volume lymph node metastasis and minor extrathyroid extension.

a high Ki-67 labeling index of $>10 \%$ and $\leq 30 \%$, which often recurs and metastasizes, and can lead to death. It corresponds to poorly differentiated carcinomas [28] (Fig. 2), thyroid carcinomas with high-grade histology [29], and cases with distant metastases [30]. The 5-year tumor-specific survival rate was estimated to be $40-80 \%$. Moderate-risk thyroid carcinoma of follicular cell origin is defined by a Ki-67 labeling index of $>5$ and $\leq 10 \%$. It is termed moderate-risk carcinoma because the value is between those of low-risk and high-risk carcinomas. It corresponds to widely invasive FTC, aggressive variant PTC, and PTC with a gross extrathyroid extension (Fig. 3). Low-risk thyroid carcinoma of follicular cell origin is a group of carcinomas that have a Ki-67 labeling index of $\leq 5 \%$ (Fig. 4), which is a very slow growth rate that overlaps significantly with those of benign 


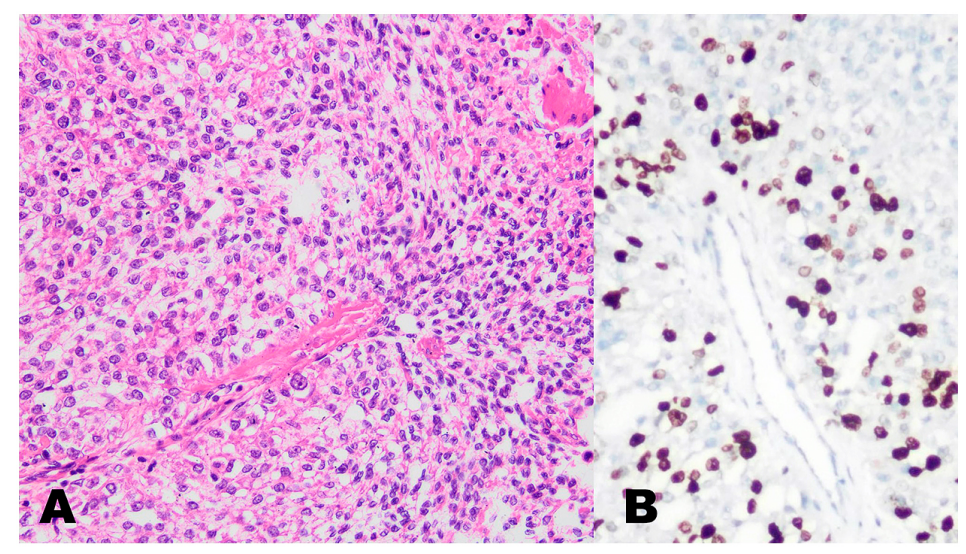

Fig. 2 An example of high-risk carcinoma of follicular cell origin found in a 56-year-old Chinese male patient who developed lung metastasis after total thyroidectomy. A: Poorly differentiated carcinoma with a solid growth pattern. Note a small focus of tumor necrosis on the upper right corner. B: Ki-67 labeling index of greater than $20 \%$. Please note the negative staining in a necrotic area at the upper right corner of B. (A: HE staining, $\times 20$, B: Ki-67 immunohistochemistry, $\times 40$ )

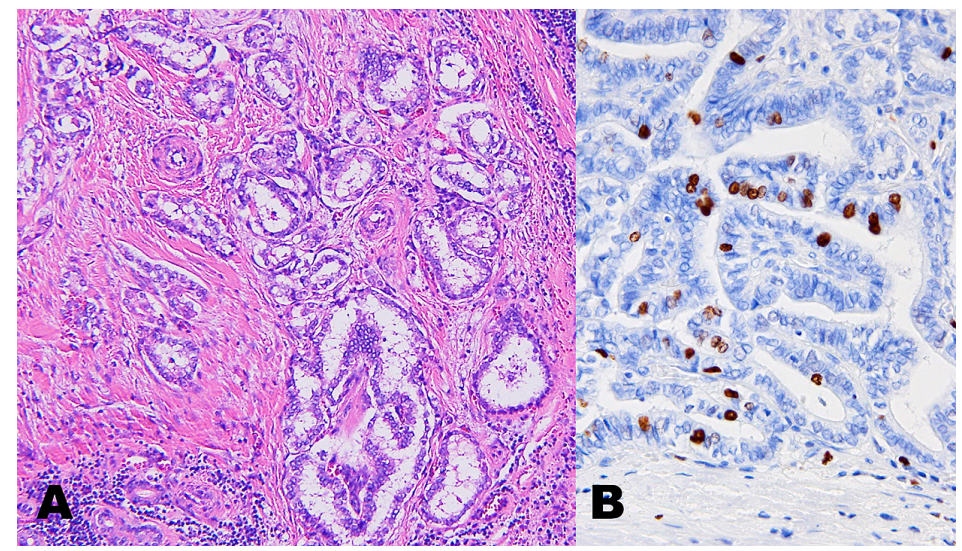

Fig. 3 An example of moderate-risk carcinoma of follicular cell origin found in a 59-year-old Japanese female. Papillary carcinoma, pT4a, Ex2 (trachea), pN1b. The patient underwent total thyroidectomy at an advanced stage with trachea invasion. After one year, she had an increased serum thyroglobulin level $(101 \mathrm{ng} / \mathrm{mL})$ and multiple lung metastases on CT examination. A: Invasive papillary carcinoma invading extrathyroid fibrous connective tissue and B: Ki-67 labeling index of approximately 9\%. (A: HE staining, $\times 10$, B: Ki-67 immunohistochemistry, $\times 20$ )

and borderline tumors (Ki-67 labeling index of $<3 \%$ ) (Fig. 5).

The first study of the Ki-67 index focusing on PTCs was by Kjellman et al., and they demonstrated an association between a high Ki-67 index and more aggressive disease (recurrence, metastasis, and cancer death) [9], which was later confirmed by several authors [11-13, 31-36]. The Ki-67 labeling index was reported to be an independent prognostic factor for recurrence $[11,24,33$, $34]$ and cancer death [11, 24]. Pan et al. and Guadagno et al. conducted meta-analyses separately. They found significant associations between the $\mathrm{Ki}-67$ labeling index and patient age, tumor size, nodal metastasis, distant metastasis, extrathyroid extension, and TNM stage. Furthermore, they confirmed that patients with a high Ki-67 labeling index had a poorer disease-free survival and increased risk of mortality than patients with a low Ki-67 labeling index [32, 36]. However, earlier studies used less sensitive immunohistochemical methods and their absolute values are no longer comparable with those in recent studies, although their conclusions are overall consistent with our conclusion that the Ki-67 examination can identify curable carcinomas as cases with a low Ki-67 index. A high Ki-67 index also accurately identifies high-grade carcinomas, such as poorly differentiated carcinomas, with a Ki-67 labeling index of $>10$ and $\leq 30 \%$. Considering this, high-risk thyroid carcinoma of follicular cell origin was listed in the 4th edition WHO classification of thyroid tumors as a synonym for poorly differentiated carcinoma [25]. 




Fig. 4 An example of low-risk thyroid carcinoma of follicular cell origin found in a 32-year-old Japanese female. A: Papillary carcinoma showing expansive growth in the left lobe of the thyroid gland. B: Classic-type papillary carcinoma exhibiting a papillary growth pattern. Note ground glass nuclei, irregular nuclear contours, and few nuclear grooves characteristic of papillary carcinoma. C: There are three brown nuclei in this field, but only one was judged as a tumor nucleus. As the other two are in the glandular space, they were judged as reactive histiocytes. The Ki-67 labeling index in this field is $<1 \%$. (A: HE staining, loupe image, B: HE stain $\times 20$, C: Ki-67 immunohistochemistry, $\times 20$ )

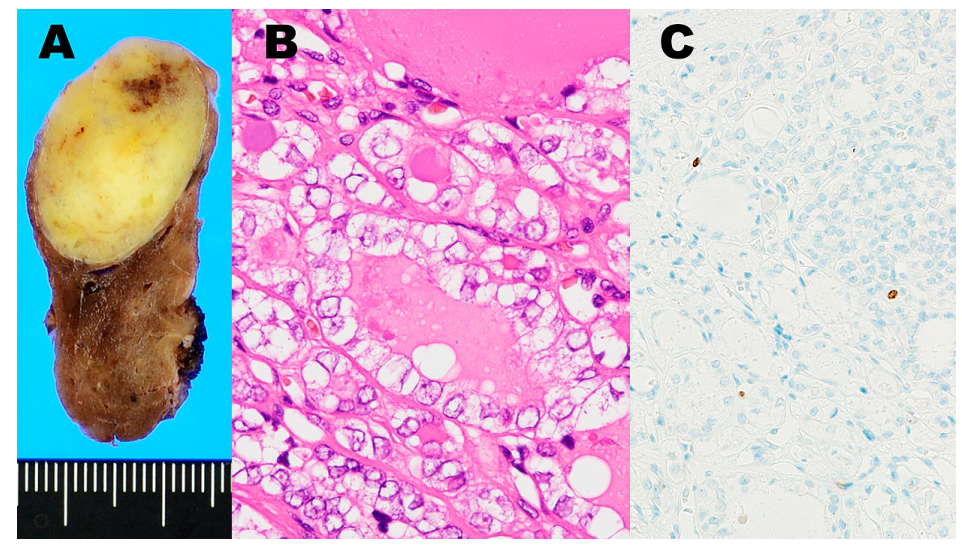

Fig. 5 An example of a borderline tumor, NIFTP (noninvasive follicular thyroid neoplasm with papillary-like nuclear features), found in a 59-year-old Japanese male patient. A: Cut surface of the thyroid demonstrates a well-demarcated solid nodule after formalin fixation. B: Higher magnification reveals nuclear irregularity and pale chromatin in tumor cells with a follicular pattern. C: Ki-67 immunohistochemistry demonstrated a low index of $<1 \%$. (A: Gross photo, B: HE staining $\times 40$, C: Ki- 67 immunohistochemistry, $\times 10$ )

\section{Patient's View}

Kitayama, a thyroid cancer survivor and one of the authors, wondered if pathologists can differentiate indolent thyroid tumors that are curable by simple excision from clinically significant cancers with a potential risk of recurrence and cancer death [37]. She believes that this distinction will help relieve the fear of recurrence because without such support, patients will fear recurrence for the rest of their lives [37]. Kitayama found many reported cases in which a complete cure was expected, even in the intermediate-risk group of PTC defined by the JAES and the ATA clinical guidelines [16, 17]. However, it required a long time and effort for her to reach this conclusion. She is concerned that many patients, who worry about recurrence and metastasis, are deprived of their peace of mind [37].

As a dermatologist, Kitayama noted the same situation in the dermatology field. There are two types of skin tumors, squamous cell carcinoma (cancer with a risk of recurrence) and basal cell carcinoma (cancer cured by simple excision). In dermatology practice, complete excision is initially performed. After excluding squamous cell carcinoma, the dermatologist usually tells the patient, "I was able to confirm that it was a basal cell carcinoma. It was completely removed and has been cured." This explanation is prepared in order for the patient to feel assured. The terms for skin cancer clearly distinguish carcinomas with a potential risk of recurrence from those cured by excision. If the pathologist makes 
this distinction clear in thyroid tumor classification, the patient will feel greater peace of mind [37]. The distinction between slow-growing cancer and very slowgrowing cancer by Welch fulfills Kitayama's request; however, Welch did not discuss how to distinguish very slow-growing cancer from slow-growing cancer clinically [1]. The risk classification using the Ki-67 labeling index, as shown in the Table 1, is the first and only histopathological classification of thyroid tumors enabling this distinction [4]. The attending physician can accurately predict the risk of recurrence and death of the patient using the Ki-67 labeling index-based classification. As most patients have very low-risk carcinoma with a Ki-67 labeling index of $\leq 5 \%$, the attending physician can relieve their anxiety immediately after surgery when they are most concerned about recurrence.

\section{Curable Thyroid Carcinomas Unlikely to Recur and Cause Death}

Ito et al. reported that the 20-year DSSR of stage I PTC was $99.3 \%$ in a Japanese patient cohort [22] even though the AJCC stage I PTC in young patients $(<55$ years old) includes a broad disease spectrum. It includes very low-risk diseases, such as intrathyroidal papillary microcarcinomas (ATA and JAES low-risk), to clinically significant carcinomas such as PTCs with $>5$ lymph node metastasis (ATA and JAES intermediate-risk) and PTCs with gross extrathyroidal extension (ATA and JAES high-risk). This suggested that there were many thyroid carcinomas with lymph node metastasis and extrathyroid extension that did not recur or metastasize. By excluding older patients ( $\geq 55$ years old) and Ki-67 moderate- and high-risk carcinomas in the young age group, the author estimated the DSSR of early-stage (T1-2 M0) PTC to approach almost $100 \%$ when curative treatment is carried out successfully. The author further proposes integrating four features, young age $(<55$ years old), early-stage (T1-2 M0), curatively treated, and low tumor growth rate (Ki-67 labeling index $\leq 5 \%$ ), to identify curable carcinomas that are unlikely to recur, metastasize, or cause cancer death. This method is almost equivalent to a strategy reported by Ghaznavi et al., which integrated three clinical features, young patients $(<55$ years old), ATA low-risk categories, and AJCC stage I [38]. They reported a DSSR of $100 \%$ for welldifferentiated thyroid carcinomas when they fulfilled these three features [38]. However, our proposed method covers more patients (both the ATA low-risk and intermediate-risk patients) than Ghaznavi's method, as there are still many curable PTCs among ATA intermediate-risk carcinomas, such as cases with regional lymph node metastasis and minor extrathyroid extension, which accounted for $38.4 \%$ of differentiated thyroid carcinomas by Grani et al., 56\% of PTCs by Ghaznavi et al. and $68.0 \%$ of PTCs by Lee et al. [38-40].

After the re-stratification based on the dynamic risk stratification system, among patients who had an initial excellent response, the probability of having structural incomplete response was reduced from 3 to $2 \%$ in ATA low-risk patients and from 18 to $2 \%$ in ATA intermediate risk patients by a thorough review by Pitoia and Jerkovich [41]. Their conclusion is in good agreement with our conclusion, that is integrating four clinical features, (young age, early-stage (T1-2 M0), curatively treated, and low Ki-67 labeling index of $<5 \%$ ), to identify very low-risk PTCs that are unlikely to recur, metastasize, or cause cancer death immediately after initial thyroid surgery.

Randolph et al. reviewed the literature and concluded that small-volume microscopic lymph node metastases in PTC are often of little clinical significance [42]. In their interpretation, PTC is often associated with subclinical microscopic lymph node metastases, and these lesions usually do not progress and seldom become clinically relevant [42]. In our interpretation, the tumor growth speed determines the clinical significance of microscopic lymph node metastases, and tumors with a high Ki-67 index develop into clinically significant diseases, whereas those with a low Ki-67 index remain stable and clinically insignificant. Midorikawa et al. observed growth arrest (self-limiting cancer) in many cases of pediatric PTC with lymph node metastases on ultrasound during clinical follow-up of histologically confirmed PTC [43]. Furthermore, several authors emphasized that minor extrathyroid extension did not impact the prognosis [44-46], and it was excluded from the T3 criteria by the 8th edition of the AJCC staging system (AJCC) [6]. Based on these observations, the author concludes that cases of small-volume lymph node metastasis and minor extrathyroid extension have an excellent prognosis as long as the Ki-67 is very low. The patient age, younger than 55 years old, was integrated into this risk classification to identify curable thyroid carcinoma with a negligible risk of recurrence, metastasis, and cancer death. It was designed to exclude ATA and JAES high-risk patients often found in older age groups; however, Ito et al. reported that the JAES low-risk patients in the older age group also had a favorable prognosis equivalent to that of the young age group [47, 48]. As this study concentrated on the young age group, future studies should focus on identifying curable PTCs in older age groups.

In summary, a Ki-67 labeling index of $\leq 5 \%$ can identify a significant number of curable carcinomas in ATA intermediate-risk and high-risk patients. This con- 
clusion is significant for clinicians to avoid the overtreatment of intermediate-risk and high-risk patients, as most clinical guidelines recommended total thyroidectomy and adjuvant RAI treatment [49-51]. Avoiding unnecessary completion thyroidectomy (aggressive cancer surgery), particularly for young patients, is essential because a significant percentage has a low Ki-67 index (Fig. 4) and an excellent prognosis. Please refer to clinical management in the Table 1, as recommended by the author. Aggressive cancer treatments (total thyroidectomy followed by RAI) should be applied only to patients with a high $(>10 \%) \mathrm{Ki}-67$ index and advanced-stage disease.

\section{Pitfalls of the Ki-67 Index}

A limitation of the Ki-67-based risk classification is the requirement of surgical specimens. Thus, the tumor growth speed is evaluable only after surgery. It is desirable to determine the growth speed before surgery to prevent unnecessary surgery for benign tumors, borderline tumors, and low-risk carcinomas [23]. Fineneedle aspiration biopsy cytology or core needle biopsy specimens may be necessary [10]. However, biopsy specimens to not always represent hot spots, suggesting that these procedures miss lesions with high proliferative activity. The second limitation is that it predicts a longterm prognosis of more than 20 years with only a single growth data point. As the proliferation capacity of thyroid carcinoma changes with age [52-59], the long-term prognosis cannot be accurately predicted when cancer progression occurs and the growth rate varies. Proper adjustment is clinically desirable when possible. Clinical methods for assessing tumor growth potential include: 1) Estimating the growth rate from size changes on tumor images (primary thyroid tumor and recurrence when clinical follow-up is selected) [43, 56-58]. 2) Measurement of the Ki-67 index using tissue specimens (primary thyroid tumors and recurrent tumors). 3) Measurement of the serum thyroglobulin doubling time and serum thyroglobulin doubling rate if the patient has recurrence/ metastasis after total thyroidectomy [43, 53, 60].

As there are several pitfalls in Ki-67 immunohistochemistry and measurement of the proliferation index, it is important to confirm that there are no conflicting clinical features when predicting the absence of recurrence, metastasis, and cancer death in patients with a Ki-67 index of $\leq 5 \%$. Ki-67 staining is negative (false negative) in specimens with tumor necrosis or low in samples with poor antigen preservation, such as decalcified specimens. On the other hand, Ki-67-positive cells may be found among non-cancer cells such as vascular endothelial cells, histiocytes, and lymphocytes; the measured value increases when erroneously counted as positive tumor cells. In addition to these technical issues, there is tumor diversity in the proliferation index, and relatively lowand high-labeled areas may coexist within a single tumor or between the primary tumor and metastatic tumor [61]. There is no consensus on which to consider as the representative value. The author is currently reporting the highest value (hotspot) as the representative value. Scoring larger numbers of tumor cells may improve the accuracy of the Ki-67 index and is desirable, but scoring all cells on a whole-tissue section is impractical for most pathologists. We recommend counting positive cells among at least 500 tumor cells in hot spot areas to assess the Ki-67 index. Measurement of the cell proliferation fraction using Ki-67 immunohistochemistry, despite these pitfalls and technical difficulties, is an inexpensive method that can be performed at all pathology laboratories worldwide, differing from costly gene panel tests. However, most Western reporting systems do not recommend the Ki-67 index in the diagnosis of thyroid tumors $[25,62]$. They emphasize increased mitotic counts and tumor necrosis (reflecting high proliferation capacity but lower sensitivity) to identify high-grade thyroid carcinomas and poorly differentiated thyroid carcinomas [25, 28, 29, 62]. However, their absence does not guarantee the indolent nature of thyroid carcinomas, as the majority ( $>95 \%)$ of PTC and FTC cases do not demonstrate increased mitoses or tumor necrosis.

\section{Message to Endocrinologists and Thyroid Surgeons}

The prognostic classification of thyroid tumors based on the Ki-67 index (Table 1) can predict the prognosis immediately after surgery. It accurately distinguishes low-risk thyroid carcinomas from moderate- and highrisk thyroid carcinomas. Advanced-stage carcinomas and non-curatively treated cases should be excluded from low-risk carcinoma of follicular cell origin, even with a Ki-67 labeling index $\leq 5 \%$. Thus, thyroid carcinomas with a low $(\leq 5 \%) \mathrm{Ki}-67$ labeling index can be further divided into two groups. 1) Curatively treated thyroid carcinomas at early-stage (T1-2 M0) for which a complete cure and long life expectancy are expected, and 2) non-curatively treated advanced stage (T3-4) thyroid carcinomas (incomplete resection) and stage II (with distant metastasis), which are persistent and have a potential risk of metastasis and cancer death. In conclusion, the author proposes integrating four clinical features, young age, early-stage (T1-2 M0), curatively treated, and low tumor growth rate (Ki-67 labeling index of $\leq 5 \%$ ), to identify very low-risk PTCs that are unlikely to recur, metastasize, or cause cancer death. For young patients with carcinoma with a low Ki-67 index after curative surgery, the 
clinician can provide significant relief after surgery if the patient fears recurrence $[63,64]$. Most PTCs have a Ki-67 labeling index of $\leq 5 \%$ based on the author's experience (Table 1). It can be further confirmed with a dynamic risk assessment proposed by Tuttle et al. and Rosario et al. in patients who achieve an excellent response (absence of elevated serum thyroglobulin or structural disease detected by imaging tests) during clinical follow-up [41, 65, 66].

Recently Odate et al. published 10 cases of PTC operated on at Ito Hospital and Yamanashi University for 40 years [67]. In these 10 cases, the tumor recurred in the lymph nodes, developed anaplastic transformation, and eight patients died of tumor [67]. The Ki-67 labeling index was low at $<5 \%$ in 8 cases of primary thyroid PTC, and it was not available in the other 2. As there were seven patients older than 55 years old and 7 subjects with advanced disease (pT3-4a), the proposed criteria successfully excluded all ten fatal PTCs from indolent thyroid tumors with an excellent prognosis.

\section{Disclosure}

All eight authors have nothing to disclose.

\section{References}

1. Welch HG, Black WC (2010) Overdiagnosis in cancer. $J$ Natl Cancer Inst 102: 605-613.

2. Davies L, Welch HG (2014) Current thyroid cancer trends in the United States. JAMA Otolaryngol Head Neck Surg 140: 317-322.

3. Li M, Maso LD, Vaccarella S (2020) Global trends in thyroid cancer incidence and the impact of overdiagnosis. Lancet Diabtes Endocrinol 8: 468-470.

4. Kakudo K, Wakasa T, Ohta Y, Yane K, Ito Y, et al. (2015) Prognostic classification of thyroid follicular cell tumors using Ki-67 labeling Index: how to report high-risk thyroid carcinomas? Endocr J 62: 1-12.

5. Bai Y, Kakudo K, Jung CK (2020) Updates in the pathologic classification of thyroid neoplasms: a review of the WHO classification. Endocr Metab (Seoul) 35: 696-715.

6. Tuttle RM, Haugen B, Perrier ND (2017) Updated American Joint Committee on Cancer/Tumor-NodeMetastasis staging system for differentiated and anaplastic thyroid cancer (eighth edition): what changed and why? Thyroid 27: 751-756.

7. Katoh R, Bray CE, Suzuki K, Komiyama A, Hemmi A, et al. (1995) Growth activity in hyperplastic and neoplastic human thyroid determined by an immunohistochemical staining procedure using monoclonal antibody MIB-1. Human Pathol 26: 139-146.

8. Tallini G, Garcia-Rostan G, Herrero A, Zelterman D, Viale G, et al. (1999) Downregulation of p27KIP1 and Ki67/Mib1 labeling index support the classification of thyroid carcinoma into prognostically relevant categories. Am J Surg Pathol 23: 678-685.

9. Kjellman P, Wallin G, Hoog A, Auer G, Larsson C, et al. (2003) MIB-1 index in thyroid tumors: a predictor of the clinical course in papillary thyroid carcinoma. Thyroid 13: 371-380.

10. Sofiadis A, Tani E, Foukakis T, Kjellman P, Skoog L, et al. (2009) Diagnostic and prognostic potential of MIB-1 proliferation index in thyroid fine needle aspiration biopsy. Int J Oncol 35: 369-374.

11. Ito Y, Miyauchi A, Kakudo K, Hirokawa M, Kobayashi $\mathrm{K}$, et al. (2010) Prognostic significance of Ki-67 labeling index in papillary thyroid carcinoma. World $J$ Surg 34: 3015-3021.

12. Miyauchi A, Kudo T, Hirokawa M, Ito Y, Kihara M, et al. (2013) Ki-67 labeling index is a predictor of postoperative persistent disease and cancer growth and a prognostic indicator in papillary thyroid carcinoma. Eur Thyroid $J 2$ : 57-64.

13. Tang J, Gui C, Qiu S, Wang M (2018) The clinicopathological significance of Ki67 in papillary thyroid carcinoma: a suitable indicator? World J Surg Oncol 16: 100.

14. Kakudo K, Bychkov A, Bai Y, Li Y, Liu Z, et al. (2018) The new 4th edition World Health Organization classification for thyroid tumors, Asian perspectives. Pathol Int 68: 641-664.

15. Goffredo P, Cheung K, Roman SA, Sosa JA (2013) Can minimally invasive follicular thyroid cancer be approached as a benign lesion?: a population-level analysis of survival among 1,200 patients. Ann Surg Oncol 20: 767-772.

16. Haugen BR, Alexander EK, Bible KC, Doherty GM, Mandel SJ, et al. (2016) American Thyroid Association management guidelines for adult patients with thyroid nodules and differentiated thyroid cancer. Thyroid 26: 1133.

17. Ito $\mathrm{Y}$, Miyauchi A, Oda H, Masuoka H, Higashiyama T, et al. (2019) Appropriateness of the revised Japanese guidelines' risk classification for the prognosis of papillary thyroid carcinoma: a retrospective analysis of 5,845 papillary thyroid carcinoma patients. Endocr $J$ 66: $127-$ 134.

18. Kim M, Kim WG, Oh HS, Park S, Kwon H, et al. (2017) Comparison of the seventh and eighth editions of the American Joint Committee on Cancer/Union for International Cancer Control tumor-node-metastasis staging system for differentiated thyroid cancer. Thyroid 27: $1149-1155$.

19. Tam S, Boonsripitayanon M, Amit M, Fellman BM, Li Y, et al. (2018) Survival in differentiated thyroid cancer: comparing the AJCC cancer staging seventh and eighth editions. Thyroid 28: 1301-1310. 
20. van Velsen EFS, Stegenga MT, van Kemenade FJ, Kam BLR, van Ginhoven TM, et al. (2018) Comparing the prognostic value of the eighth edition of the American Joint Committee on Cancer/Tumor Node Metastasis staging system between papillary and follicular thyroid carcinoma. Thyroid 28: 976-981.

21. Nixon IJ, Wang LY, Migliacci JC, Eskander A, Campbell MJ, et al. (2016) An international multi-institutional validation of age 55 years as a cutoff for risk stratification in the AJCC/UICC staging system for well-differentiated thyroid cancer. Thyroid 26: 373-380.

22. Ito Y, Miyauchi A, Hirokawa M, Yamamoto M, Oda H, et al. (2018) Prognostic value of the 8 th edition of the tumornode metastasis classification for patients with papillary thyroid carcinoma: a single-institution study at a high volume center in Japan. Endocr J 65: 707-716.

23. (2020) National Cancer Institute dictionary of cancer terms. https://www.cancer.gov/publications/dictionaries/ cancer-terms/def/cancer accessed on December 31, 2020.

24. Scholzen T, Gerdes J (2000) The ki-67 protein: from the known and the unknown. J Cell Physiol 182: 311-322.

25. Lloyd RV, Osamura RY, Klöppel G, Rosai J (ed) (2017) WHO Classification of Tumours of Endocrine Organs (4th). IARC, Lyon, France.

26. Hirokawa M, Sugitani I, Kakudo K, Sakamoto A, Higashiyama T, et al. (2016) Histopathological analysis of anaplastic thyroid carcinoma cases with long-term survival: a report from the Anaplastic Thyroid Carcinoma Research Consortium of Japan. Endocr J 63: 441-447.

27. Kakudo K, Asai M, Bai Y (2017) How to confirm or deny high-risk thyroid carcinoma is a challenge for pathologists. Pathol Int 67: 173-180.

28. Volante M, Collini P, Nikiforov YE, Sakamoto A, Kakudo $\mathrm{K}$, et al. (2007) Poorly differentiated thyroid carcinoma: the Turin proposal for the use of uniform diagnostic criteria and an algorithmic diagnostic approach. Am J Surg Pathol 31: 1256-1264.

29. Gnemmi V, Renaud F, Do Cao C, Salleron J, Lion G, et al. (2014) Poorly differentiated thyroid carcinomas: application of the Turin proposal provides prognostic results similar to those from the assessment of high-grade features. Histopathology 64: 263-273.

30. Durante C, Haddy N, Baudin E, Leboulleux S, Hartl D, et al. (2006) Long-term outcome of 444 patients with distant metastases from papillary and follicular thyroid carcinoma: benefits and limits of radioiodine therapy. J Clin Endocrinol Metab 91: 2892-2899.

31. Mussig K, Wehrmann T, Dittmann H, Wehrmann M, Ueberberg B, et al. (2012) Expression of the proliferation marker Ki-67 associates with tumour staging and clinical outcome in differentiated thyroid carcinomas. Clin Endocrinol (Oxf) 77: 139-145.

32. Pan DH, Wen DY, Luo YH, Chen G, Yang H, et al. (2017) The diagnostic and prognostic values of Ki-67/MIB-1 expression in thyroid cancer: a meta-analysis with 6051 cases. Onco Targets Ther 10: 3261-3276.

33. Yao X, Meng Y, Guo R, Lu G, Jin L, et al. (2020) Value of ultrasound combined with immunohistochemistry evaluation of central lymph node metastasis for the prognosis of papillary thyroid carcinoma. Cancer Manag Res 12: 8787-8799.

34. Viana AOR, Gonçalves Filho J, Francisco ALN, Pinto CAL, Kowalski LP (2020) Ki-67 and CK-19 are predictors of locoregional recurrence in papillary thyroid carcinoma. Acta Otorhinolaryngol Ital 40: 190-197.

35. Avdalyan AM, Ivanov AA, Lushnikova EL, Molodykh OP, Vikhlyanov IV (2020) The relationship of immunoexpression of Ki-67 and Hsp70 with clinical and morphological parameters and prognosis of papillary thyroid cancer. Bull Exp Biol Med 168: 688-693.

36. Guadagno E, D'Avella E, Cappabianca P, Colao A, Del Basso De Caro M (2020) Ki67 in endocrine neoplasms: to count or not to count, this is the question! A systematic review from the English language literature. $J$ Endocr Invest 43: 1429-1445.

37. (2020) Kitayama N, Kakudo K: A doctor becomes a patient, thyroid cancer. http://www.med.osaka-u.ac.jp/pub/ labo/JCJTC/EE1.html accessed on December 31, 2020.

38. Ghaznavi SA, Ganly I, Shaha AR, English C, Wills J, et al. (2018) Using the American Thyroid Association risk-stratification system to refine and individualize the American Joint Committee on Cancer eighth edition disease-specific survival estimates in differentiated thyroid cancer. Thyroid 28: 1293-1300.

39. Lee SG, Lee WK, Lee HS, Moon J, Lee CR, et al. (2017) Practical performance of the 2015 American Thyroid Association Guidelines for predicting tumor recurrence in patients with papillary thyroid cancer in South Korea. Thyroid 27: 174-181.

40. Grani G, Zatelli MC Alfo M, Montesano T, Torlontano M, et al. (2021) Real-world performance of the American Thyroid Association risk estimates in predicting 1-year differentiated thyroid cancer outcomes: a prospective multicenter study of 2000 patients. Thyroid 31: 264-271.

41. Pitoia F, Jerkovich F (2019) Dynamic risk assessment in patients with differentiated thyroid cancer. Endocr Relat Cancer 26: R553-R566.

42. Randolph GW, Duh QY, Heller KS, LiVolsi VA, Mandel SJ, et al. (2012) The prognostic significance of nodal metastases from papillary thyroid carcinoma can be stratified based on the size and number of metastatic lymph nodes, as well as the presence of extranodal extension. Thyroid 22: 1144-1152.

43. Midorikawa S, Ohtsuru A, Murakami M, Takahashi H, Suzuki S, et al. (2018) Comparative analysis of the growth pattern of thyroid cancer in young patients screened by ultrasonography in Japan after a nuclear accident. The Fukushima Health Management Survey. JAMA Otolaryngol Head Neck Surg 144: 57-63.

44. Ito Y, Tomoda C, Uruno T, Takamura Y, Miya A, et al. (2006) Prognostic significance of extrathyroid extension of papillary thyroid carcinoma: massive but not minimal extension affects the relapse-free survival. World J Surg 30: $780-786$. 
45. Hay ID, Johnson TR, Thompson GB, Sebo TJ, Reinalda MS (2016) Minimal extrathyroid extension in papillary thyroid carcinoma does not result in increased rates of either cause-specific mortality or postoperative tumor recurrence. Surgery 159: 11-21.

46. Li G, Li R, Song L, Chen W, Jiang K, et al. (2020) Implications of extrathyroidal extension invading only the strap muscles in papillary thyroid carcinomas. Thyroid 30: $57-$ 64.

47. Ito $\mathrm{Y}$, Miyauchi A, Yamamoto M, Masuoka H, Higashiyama T, et al. (2020) Subset analysis of the Japanese risk classification guidelines for papillary thyroid carcinoma. Endocr J 67: 275-282.

48. Ito Y, Miyauchi A, Higashiyama T, Masuoka H, Kihara M, et al. (2020) Older age significantly affects mortality of patients with papillary thyroid carcinoma only when they have high-risk features. World J Surg 44: 1885-1891.

49. Cooper DS, Doherty GM, Haugen BR, Kloos RT, Lee SL, et al. (2009) Revised American Thyroid Association management guidelines for patients with thyroid nodules and differentiated thyroid cancer. Thyroid 19: 1167-1214.

50. Haugen BR, Sawka AM, Alexander EK, Bible KC, Caturegli P, et al. (2017) American Thyroid Association guidelines on the management of thyroid nodules and differentiated thyroid cancer task force review and recommendation on the proposed renaming of encapsulated follicular variant papillary thyroid carcinoma without invasion to noninvasive follicular thyroid neoplasm with papillary-like nuclear features. Thyroid 27: 481-483.

51. Ferris RL, Nikiforov Y, Terris D, Seethala RR, Ridge JA, et al. (2018) AHNS series: do you know your guidelines? AHNS endocrine section consensus statement: state-ofthe-art thyroid surgical recommendations in the era of noninvasive follicular thyroid neoplasm with papillarylike nuclear features. Head Neck 40: 1881-1888.

52. Saad AG, Kumar S, Ron E, Lubin JH, Stanek J, et al. (2006) Proliferative activity of human thyroid cells in various age groups and its correlation with the risk of thyroid cancer after radiation exposure. J Clin Endocrnol Metab 91: 2672-2677.

53. Miyauchi A, Kudo T, Kihara M, Higashiyama T, Ito Y, et al. (2013) Relationship of biochemically persistent disease and thyroglobulin-doubling time to age at surgery in patients with papillary thyroid carcinoma. Endocr $J$ 60: 415-421.

54. Ito Y, Miyauchi A, Kobayashi K, Miya A (2014) Prognosis and growth activity depend on patient age in clinical and subclinical papillary thyroid carcinoma. Endocr $J 61$ : 205-213.

55. Takano T (2017) Natural history of thyroid cancer. Endocr J 64: 237-244.

56. Miyauchi A, Kudo T, Ito Y, Oda H, Sasai H, et al. (2018) Estimation of the lifetime probability of disease progression of papillary microcarcinoma of the thyroid during active surveillance. Surgery 163: 48-52.

57. Miyauchi A, Kudo T, Ito Y, Oda H, Yamamoto M, et al. (2019) Natural history of papillary thyroid microcarcinoma: kinetic analyses on tumor volume during active surveillance and before presentation. Surgery 165: 25-30.

58. Kasahara T, Miyauchi A, Ito Y, Kudo T, Masuoka H, et al. (2020) Tumor volume kinetic analyses might explain excellent prognoses in young patients with papillary thyroid carcinoma. J Thyroid Res 2020: 4652767.

59. Takano T (2020) Overdiagnosis of juvenile thyroid cancer: time to consider self-limiting cancer. J Adolesc Young Adult Oncol 9: 286-288.

60. Koshkina A, Fazelzad R, Sugitani I, Miyauchi A, Thabane $\mathrm{L}$, et al. (2020) Association of patient age with progression of low-risk papillary thyroid carcinoma under active surveillance: a systematic review and meta-analysis. JAMA Otolaryngol Head Neck Surg 146: 552-560.

61. Polley MYC, Leung SCY, MacShane LM, Gao D, Hugh JC, et al. (2013) An international Ki67 reproducibility study. J Natl Cancer Inst 105: 1897-1906.

62. Ghossein R, Barletta JA, Bullock M, Johnson SJ, Kakudo $\mathrm{K}$, et al. (2021) Data set for reporting carcinoma of the thyroid: recommendations from the International Collaboration on Cancer Reporting. Human Pathol 110: 62-72.

63. Hedman C, Djarv T, Strang P, Lundgren CI (2018) Fear of recurrence and view of life affect health-related quality of life in patients with differentiated thyroid carcinoma: a prospective Swedish population-based study. Thyroid 28: 1595-1608.

64. Pitt SC, Saucke MC, Wendit EM, Schneider DF, Orne J, et al. (2020) Patients' reaction to diagnosis with thyroid cancer or an indeterminate thyroid nodule. Thyroid 31: 19.

65. Tuttle RM, Tala H, Shah J, Leboeuf R, Ghossein R, et al. (2010) Estimating risk of recurrence in differentiated thyroid cancer after total thyroidectomy and radioactive iodine remnant ablation: using response to therapy variables to modify the initial risk estimates predicted by the new American Thyroid Association staging system. Thyroid 20: 1341-1349.

66. Rosario PW, Furtado MS, Mourao GF, Calsolari MR (2015) Patients with papillary thyroid carcinoma at intermediate risk of recurrence according to American Thyroid Association criteria can be reclassified as low risk when the postoperative thyroglobulin is low. Thyroid 25: 12431248.

67. Odate T, Oishi N, Kawai M, Tahara I, Mochizuki K, et al. (2021) Progression of papillary thyroid carcinoma to anaplastic carcinoma in metastatic lymph nodes: solid/insular growth and hobnail cell change in lymph nodes are predictors of subsequent anaplastic transformation. Endocr Pathol. 2021 Mar 24. doi: 10.1007/s12022-021-09674-1. Online ahead of print. 